\title{
ON THE RELATIONSHIP AMONG THE DIAGONAL FILES OF A PADÉ TABLE*
}

\author{
BY H. S. WALL
}

1. Introduction. The object of the following note is to investigate the relationship among the $n$th approximants of the different diagonal files of a Padé table; and to study the relationship among the limits of those files for a Stieltjes power series, in the case $\dagger$ that those files have different limits. We have found that an arbitrary file $S_{k}$ converges to an expression of the form

$$
\frac{\alpha_{k} p-\beta_{k} p_{1}}{\alpha_{k} q-\beta_{k} q_{1}}
$$

where $p, p_{1}, q, q_{1}$ are entire transcendental functions independent of $k$, and $\alpha_{k}, \beta_{k}$ are polynomials or constants. If we denote by $u_{k}, v_{k}$ the numerator and denominator, respectively, of (1), then if $k^{\prime}, k^{\prime \prime}$ are two values of the index $k$, the following identity obtains :

$$
u_{k^{\prime}, v^{\prime \prime}}-u_{k^{\prime}} v_{k^{\prime}}=\alpha_{k^{\prime}} \beta_{k^{\prime \prime}}-\alpha_{k^{\prime}} \beta_{k^{\prime}}
$$

and the polynomial on the right is not identically zero if $k^{\prime} \neq k^{\prime \prime}$.

2. Preliminary Formulas. $\ddagger$ Let $\mathfrak{B}(x)=\sum_{v=0}^{\infty} c_{v}(-x)^{v}$ be a normal power series, and let $\mathbb{E}(x)=\sum_{v=0}^{\infty} d_{v}(-x)^{v}$ be the reciprocal of $\mathfrak{P}(x)$. Set $\mathfrak{P}^{(k)}(x)=\sum_{v=0}^{\infty} c_{v+k}(-x)^{v}$, $\mathbb{E}^{(k)}(x)=\sum_{v=0}^{\infty} d_{v+k}(-x)^{v}$, $k=0,1,2, \cdots$. Then the series $\mathfrak{B}^{(k)}(x)$, $\mathfrak{E}^{(k)}(x)$ have corresponding continued fractions

$$
\frac{1}{a_{1}{ }^{(k)}}+\frac{x}{a_{2}{ }^{(k)}}+\frac{x}{a_{3}{ }^{(k)}}+\cdots, \frac{1}{b_{1}{ }^{(k)}}+\frac{x}{b_{2}{ }^{(k)}}+\frac{x}{b_{3}{ }^{(k)}}+\cdots,
$$

respectively, where the numbers $a_{n}^{(k)}, b_{n}^{(k)}$ are different from 0 .

* Presented to the Society, September 12, 1930.

$\dagger$ Designated as "Case I" in the writer's paper, On the Padé approximants associated with the continued fraction and series of Stieltjes, Transactions of this Society, vol. 31 (1929), pp. 91-116. We show in the present article that no two of the diagonal files have the same limit, thus supplementing the earlier result.

$\ddagger$ For details concerning the statements in this paragraph, see a paper by the writer in the Transactions of this Society, vol. 33 (1931), pp. 511-532. 
The Padé approximants for $\mathfrak{P}(x)$ may be expressed in terms of the numerators and denominators of the $n$th convergents, $A_{n}^{(k)} / B_{n}^{(k)}, C_{n}^{(k)} / D_{n}^{(k)}$, of these continued fractions. Indeed if $[p, q] \equiv N_{p, q} / D_{p, q}$ is that Padé approximant in which the degrees of numerator and denominator do not exceed $q$ and $p$, respectively, then we have the following formulas:

$$
\begin{aligned}
& {[n-1, n+k-1]=} \mathfrak{P}_{k}+(-x)^{k} A_{2 n-1}^{(k)} / B_{2 n-1}^{(k)}, \\
& {[n+k-1, n-1]=\left[\mathfrak{\xi}_{k}+(-x)^{k} C_{2 n-1}^{(k)} / D_{2 n-1}^{(k)}\right]^{-1}, } \\
& \quad(n-1, k=0,1,2, \cdots),
\end{aligned}
$$

where $\mathfrak{P}_{k}=\sum_{v=0}^{k-1} c_{v}(-x)^{v}, \mathfrak{F}_{k}=\sum_{v=0}^{k-1} d_{v}(-x)^{v}, \mathfrak{B}_{0}=\mathfrak{E}_{0}=0$;

$$
\begin{aligned}
{[n, n-1]=A_{2 n} / B_{2 n}, \quad[n-1, n] } & =D_{2 n} / C_{2 n}, \\
& (n=1,2,3, \cdots) .
\end{aligned}
$$

In the right member of (3) there occur the polynomials $A_{2 n-1}^{(k)}, B_{2 n-1}^{(k)}$. In what follows we shall want to express these polynomials in terms of the polynomials $A_{m}, B_{m}$. For that purpose we have the following identities which we gave in the paper to which we referred at the beginning of this paragraph, namely

$$
B_{2 n-1}^{(k)}=h_{n}^{(k-1)} B_{2 n}^{(k-1)}-B_{2 n+1}^{(k-1)},
$$

$x A_{2 n-1}^{(k)}=c_{k-1}\left(h_{n}^{(k-1)} B_{2 n}^{(k-1)}-B_{2 n+1}^{(k-1)}\right)-\left(h_{n}^{(k-1)} A_{2 n}^{(k-1)}-A_{2 n+1}^{(k-1)}\right.$, $h_{n}^{(k-1)} B_{2 n}^{(k)}=B_{2 n+1}^{(k-1)}$,

$$
x h_{n}^{(k-1)} A_{2 n}^{(k)}=c_{k-1} B_{2 n+1}^{(k-1)}-A_{2 n+1}^{(k-1)}
$$

$(n, k=1,2,3, \cdots)$. Here $h_{n}^{(k-1)}=a_{1}^{(k-1)}+a_{3}^{(k-1)}+\cdots+a_{2 n+1}^{(k-1)}$, and is $\neq 0$. There are four similar relations for the $C_{m}^{(k)}, D_{m}^{(k)}$ of (4).

3. The Diagonal Files $S_{k}, k>0$. We shall now turn to the Padé table, which is a table of double entry containing the approximant $[p, q]$ in the $(p+1)$ th row and $(q+1)$ th column. The fractions $A_{1} / B_{1}, A_{3} / B_{3}, A_{5} / B_{5}, \cdots$ constitute the principal diagonal file $S_{0}$, while $S_{-1}$, the first parallel file below $S_{0}$, is made up of the sequence $A_{2} / B_{2}, A_{4} / B_{4}, A_{6} / B_{6}, \cdots$. If $k$ is any integer, then the diagonal file $S_{k}$ is the sequence

$S_{k}:[m, m+k],\left\{\begin{array}{l}m=0,1,2, \cdots, \text { if } k \geqq 0, \\ m=-k,-k+1,-k+2, \cdots, \text { if } k<0 .\end{array}\right.$ 
The formulas (6) and (3) will enable us to express the approximants of a file $S_{k}, k>0$, in terms of the numerators and denominators of the fractions $A_{m} / B_{m}$ of the files $S_{0}$ and $S_{-1}$. Thus if we set $k=1$ in the first two formulas of (6), we have, by (3),

$$
[n-1, n]=\frac{h_{n} A_{2 n}-A_{2 n+1}}{h_{n} B_{2 n}-B_{2 n+1}} .
$$

This is the $n$th approximant in the file $S_{1}$. Setting $k$ successively equal to 1,2 in (6), we may now express $A_{2 n-1}^{\prime \prime}, B_{2 n-1}^{\prime \prime}$ in terms of the $A_{m}, B_{m}$. Then by (3) with $k=2$, we find that the $n$th approximant in the file $S_{2}$ is

$$
[n-1, n+1]=\frac{\left[\left(h_{n}^{\prime} / h_{n}\right)+x\right] A_{2 n+1}-h_{n} A_{2 n+2}}{\left[\left(h_{n}^{\prime} / h_{n}\right)+x\right] B_{2 n+1}-h_{n} B_{2 n+2}} .
$$

In the final step of the reduction we used the identities

(9) $A_{2 n+1}=a_{2 n+1} A_{2 n}+x A_{2 n-1}, B_{2 n+1}=a_{2 n+1} B_{2 n}+x B_{2 n-1}$.

To obtain the general formula, suppose that, for a particular value of $k$,

$$
[n-1, n+k-1]=\frac{M_{k} A_{2 n+k-1}-N_{k} A_{2 n+k}}{M_{k} B_{2 n+k-1}-N_{k} B_{2 n+k}},
$$

where $M_{k}, N_{k}$ are polynomials in $x$ in which the coefficients are rational functions of the quantities $h_{r}^{s}$. Let $\mathscr{X}_{k}, \mathcal{N}_{k}$ denote the polynomials obtained from these by replacing $h_{r}^{s}$ by $h_{r}^{s+1}$ throughout. Then*

$$
[n-1, n+k]=c_{0}-x \frac{\mathcal{X}_{k} A_{2 n+k-1}^{\prime}-\mathcal{N}_{k} A_{2 n+k}^{\prime}}{\mathcal{X}_{k} B_{2 n+k-1}^{\prime}-\mathcal{N}_{k} B_{2 n+k}^{\prime}} .
$$

But when $k=2 p$ this reduces, with the aid of (6), to (10) with $k=2 p+1$, where

$$
M_{2 p+1}=\mathcal{X}_{2 p} h_{n+p}, \quad N_{2 p+1}=\mathcal{X}_{2 p}+\left(\mathcal{N}_{2 p} / h_{n+p}\right) ;
$$

* This follows from the fact that the right member of (11) is a rational function of $x$ in which the degrees of numerator and denominator do not exceed $n+k$ and $n-1$, respectively; and the expansion in ascending powers of $x$ agrees with $\mathfrak{P}(x)$ for the first $2 n+k-1$ terms. See Perron, Die Lehre von den Kettenbrüchen, Chapter $\mathrm{X}$. 
and when $k=2 p-1$, (11) reduces to (10) with $k=2 p$, where

$$
M_{2 p}=\left(\mathcal{X}_{2 p-1} / h_{n+p-1}\right)+x \mathcal{N}_{2 p-1}, \quad N_{2 p}=\mathcal{N}_{2 p-1} h_{n+p-1} .
$$

By (12) and (13) we see that $M_{k+1}$ and $N_{k+1}$ are polynomials of the same character as $M_{k}$ and $N_{k}$, namely, they are polynomials in $x$ in which the coefficients are rational functions of the quantities $h_{r}{ }^{s}$. But this has been verified for small values of $k$, and is therefore universally true. Formulas (12) and (13), with the initial values obtainable from (7) and (8), may be used to compute successively the polynomials $M_{k}$ and $N_{k}$

4. The Diagonal Files $S_{-k}, k>1$. We shall next show that the approximants of the files $S_{-k}, k>1$, can be expressed in the form (10). We begin with the relations

$$
\begin{aligned}
& \mathfrak{E}(x) \sim \frac{1}{b_{1}}+\frac{x}{b_{2}}+\frac{x}{b_{3}}+\ldots \\
& \mathfrak{P}(x)=\frac{1}{\mathfrak{E}(x)} \sim b_{1}-\frac{x}{\left(-b_{2}\right)}+\frac{x}{\left(-b_{3}\right)}+\overline{\left(-b_{4}\right)}+\ldots,
\end{aligned}
$$

from which it follows that

$$
b_{2 n}=-a_{2 n-1}^{\prime}, \quad b_{2 n+1}=-a_{2 n}^{\prime}, \quad b_{1}=c_{0} .
$$

Also by symmetry we have

$$
a_{2 n}=-b_{2 n-1}^{\prime}, \quad a_{2 n+1}=-b_{2 n}^{\prime}, \quad a_{1}=d_{0} .
$$

Furthermore

$$
c_{0}-x A_{n}^{\prime} / B_{n}^{\prime}=D_{n+1} / C_{n+1},
$$

and consequently

$$
c_{0} B_{n}^{\prime}-x A_{n}^{\prime}=\gamma_{n} D_{n+1}, B_{n}^{\prime}=\gamma_{n} C_{n+1},
$$

where $\gamma_{n}$ is independent of $x$. Giving to $n$ the values $2 p$ and $2 p-1$ and employing (6) we then have

$$
\begin{aligned}
A_{2 p+1} / h_{p} & =\gamma_{2 p} D_{2 p+1}, B_{2 p+1} / h_{p}=\gamma_{2 p} C_{2 p+1}, \\
h_{p} A_{2 p}-A_{2 p+1} & =\gamma_{2 p-1} D_{2 p}, h_{p} B_{2 p}-B_{2 p+1}=\gamma_{2 p-1} C_{2 p} .
\end{aligned}
$$

For the factors $\gamma_{n}$ we have the following values:

$$
\gamma_{2 p}=1, \gamma_{2 p-1}=-1 \text {. }
$$


Indeed one may verify directly that the coefficients of the highest powers of $x$ in $A_{2 p+1}$ and $D_{2 p+1}$ are 1 and $g_{p}=b_{1}+b_{3}+b_{5}$ $+\cdots+b_{2 p+1}$, respectively. Hence by (14)

$$
1 / h_{p}=\gamma_{2 p} g_{p}=\gamma_{2 p}\left(c_{0}-a_{2}^{\prime}-a_{4}^{\prime}-\cdots-a_{2 p}^{\prime}\right) .
$$

But if we take $k=1$ in the first of the following known relations*

$$
\begin{aligned}
a_{2 n}^{(k)}=a_{2 n+1}^{(k-1)} / h_{n-1}^{(k-1)} h_{n}^{(k-1)}, a_{2 n+1}^{(k)}=a_{2 n+2}^{(k-1)}\left(h_{n}^{(k-1)}\right)^{2}, \\
\left(k=1,2, \cdots ; a_{n}{ }^{0}=a_{n}\right),
\end{aligned}
$$

this reduces to $1 / h_{p}=\gamma_{2 p} / h_{p}$, so that $\gamma_{2 p}=1$. In like manner we find that $\gamma_{2 p-1}=-1$.

Let us now put $k=2$ in (4). Then as in (8) we find that, if we set $g_{n}^{(k)}=b_{1}^{(k)}+b_{3}^{(k)}+b_{5}^{(k)}+\cdots+b_{2 n+1}^{(k)}$,

$$
[n+1, n-1]=\frac{\left[\left(g_{n}^{\prime} / g_{n}\right)+x\right] D_{2 n+1}-g_{n} D_{2 n+2}}{\left[\left(g_{n}^{\prime} / g_{n}\right)+x\right] C_{2 n+1}-g_{n} C_{2 n+2}} .
$$

But by (16) and (17), followed by an easy reduction in which the formulas (9), (14), and (15) play a part, this becomes

$$
[n+1, n-1]=\left(g_{n}^{\prime} A_{2 n+1}+A_{2 n+2}\right) /\left(g_{n}^{\prime} B_{2 n+1}+B_{2 n+2}\right),
$$

which is the $n$th approximant in $S_{-2}$.

To obtain the general formula, we apply (10) to the series $\mathfrak{E}$, and use (4). This gives a relation of the form

$$
[n+k-1, n-1]=\frac{V_{k} D_{2 n+k-1}-W_{k} D_{2 n+k}}{V_{k} C_{2 n+k-1}-W_{k} C_{2 n+k}},
$$

where the $V_{k}, W_{k}$ are polynomials in $x$ with coefficients which are rational functions of the quantities $g_{r}^{s}$, and which may be calculated by means of formulas analogous to (12) and (13). By (16) and (17), we then have

$$
[n+k-1, n-1]=\frac{M_{-k} A_{2 n+k-1}-N_{-k} A_{2 n+k}}{M_{-k} B_{2 n+k-1}-N_{-k} B_{2 n+k}},
$$

where

$$
\begin{aligned}
& M_{-2 p}=g_{n+p-1} V_{2 p}-x W_{2 p}, N_{-2 p}=-\left(W_{2 p} / g_{n+p-1}\right), \\
& M_{-2 p-1}=-V_{2 p+1} / g_{n+p}, N_{-2 p-1}=g_{n+p} W_{2 p+1}-V_{2 p+1} .
\end{aligned}
$$

* Wall, Transactions of this Society, vol. 31 (1929), pp. 102-103. 
The quantities $M_{-k}$ and $N_{-k}$ are polynomials in $x$ with coefficients which are rational functions of the numbers $g_{r}^{s}$.

The recursion formulas analogous to (12) and (13) for the $V_{k}$ and $W_{k}$ may be combined with (21) to give the following recursion formulas for the polynomials $M_{-k}, N_{-k}$ :

$$
\begin{gathered}
M_{-2 p}=-g_{n+p-1}^{\prime} \mathcal{X}_{-2 p+1}, \quad N_{-2 p}=\mathscr{X}_{-2 p+1}-\frac{\mathcal{N}_{-2 p+1}}{g_{n+p-1}^{\prime}}, \\
M_{-2 p-1}=-\frac{\mathcal{X}_{-2 p}}{g_{n+p-1}^{\prime}}+x \mathcal{N}_{-2 p}, \quad N_{-2 p-1}=-g_{n+p-1}^{\prime} \mathcal{N}_{-2 p} .
\end{gathered}
$$

Here the script letters have the same significance as before, namely $\mathfrak{X}_{-k}, \mathcal{N}_{-k}$ are polynomials obtained by replacing $g_{r}^{s}$ by $g_{r}^{s+1}$ in the corresponding polynomials $M_{-k}, N_{-k}$.

5. The Series of Stieltjes. We shall now apply the work of the preceding paragraphs to the series of Stieltjes. If we set $x=1 / z$ in $\mathfrak{P}(x)$ and in the corresponding continued fraction, and then divide by $z$, we will obtain the series

$$
\frac{c_{0}}{z}-\frac{c_{1}}{z^{2}}+\frac{c_{2}}{z^{3}}-\cdots,
$$

and continued fraction

$$
\frac{1}{a_{1} z}+\frac{1}{a_{\Omega}}+\frac{1}{a_{3} z}+\cdots .
$$

These are the forms of the series and continued fraction found in Stieltjes' Recherches sur les fractions continues. ${ }^{*}$

The numerators and denominators, $P_{n}(z)$ and $Q_{n}(z)$, of the $n$th convergents of (24) are connected with the $A_{n}(x), B_{n}(x)$ by the following equations:

$$
\begin{aligned}
P_{2 n}(z) & =z^{n-1} A_{2 n}(1 / z), \quad Q_{2 n}(z)=z^{n} B_{2 n}(1 / z), \\
P_{2 n+1}(z) & =z^{n} A_{2 n+1}(1 / z), Q_{2 n+1}(z)=z^{n+1} B_{2 n+1}(1 / z)
\end{aligned}
$$

When $a_{n}>0$, and $\sum a_{n}$ is convergent, Stieltjes found that

$$
\begin{array}{ll}
\lim _{n} P_{2 n}(z)=p(z), & \lim _{n} Q_{2 n}(z)=q(z), \\
\lim _{n} P_{2 n+1}(z)=p_{1}(z), & \lim _{n} Q_{2 n+1}(z)=q_{1}(z),
\end{array}
$$

* Stieltjes, Oeuvres, vol. 2. 
where $p(z), p_{1}(z), q(z), q_{1}(z)$ are entire transcendental functions of genre 0 , connected by the relation

$$
p_{1}(z) q(z)-p(z) q_{1}(z)=+1 .
$$

Let us replace $x$ by $1 / z$ in (10) and (20), divide by $z$, and then introduce the polynomials $P_{n}(z)$ and $Q_{n}(z)$ of (25). After removing a common power of $1 / z$ from numerators and denominators, these expressions then take the form

$$
\frac{G_{k} P_{\delta-1}-H_{k} P_{\delta}}{G_{k} Q_{\delta-1}-H_{k} Q_{\delta}}
$$

where $\delta=2 n+|k|,(k=0, \pm 1, \pm 2, \cdots)$. Here the $G_{k}, H_{k}$ are polynomials in $z$ given in terms of the $M_{k}, N_{k}$ by the equations

$$
\begin{aligned}
G_{k}(z)= & z^{\theta} M_{k}(1 / z), H_{k}=z^{\theta^{\prime}} N_{k}(1 / z), \\
& \theta^{\prime}=\theta-\left[1-(-1)^{k}\right] / 2,
\end{aligned}
$$

where $\theta$ is the larger of the degrees of $M_{k}(x), N_{k}(x)$ if $k$ is even, and of $M_{k}(x), x N_{k}(x)$ if $k$ is odd. In particular, we have

$$
G_{0}=1, H_{0}=0, G_{-1}=1, H_{-1}=0 .
$$

The others may be calculated successively by means of the recursion formulas (12), (13), (22). For example, we find that

$$
\begin{aligned}
G_{1} & =h_{n} z, H_{1}=1 ; G_{2}=\left(h_{n}^{\prime} / h_{n}\right) z+1, H_{2}=h_{n} z, \\
G_{3} & =\left(h_{n}^{\prime \prime} h_{n+1} / h_{n}^{\prime}\right) z^{2}+h_{n+1} z, \\
H_{3} & =\left[\left(h_{n}^{\prime \prime} / h_{n}^{\prime}\right)+\left(h_{n}^{\prime} / h_{n+1}\right)\right] z+1, \\
G_{-2} & =g_{n}^{\prime}, H_{-2}=-1 ; G_{-3}=-\left(g_{n}^{\prime \prime} / g_{n}^{\prime}\right) z-1, H_{-3}=g_{n}^{\prime} .
\end{aligned}
$$

It is seen that in these cases

$$
\begin{aligned}
& G_{2 p}(0)=1, \quad H_{2 p}(0)=0,
\end{aligned}
$$

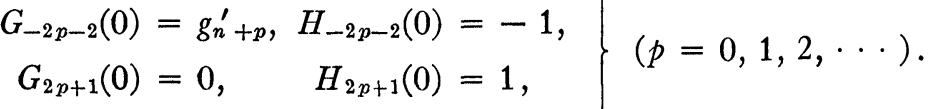

$$
\begin{aligned}
& G_{-2 p-3}(0)=-1, \quad H_{-2 p-3}(0)=g_{n+p}^{\prime} .
\end{aligned}
$$

The proof of (32) for all $p$ is readily accomplished by mathematical induction.

Now when $a_{n}>0$ and $\sum a_{n}$ converges, we see by (18) that $a_{n}^{\prime}>0$ and $\sum a_{n}^{\prime}$ converges. Applying (18) again we find that 
$a_{n}{ }^{\prime \prime}>0$ and $\sum a_{n}{ }^{\prime \prime}$ converges, etc. Also by (15) $b_{n}^{\prime}<0$ and $\sum b_{n}^{\prime}$ converges. Then by (18) with $k=2$ and $a_{n}^{\prime}, a_{n}{ }^{\prime \prime}$ replaced by $b_{n}{ }^{\prime}$ and $b_{n}{ }^{\prime \prime}$, respectively, it follows that $b_{n}{ }^{\prime \prime}<0$ and $\sum b_{n}{ }^{\prime \prime}$ converges, etc. Continuing in this way we find that all the series $\sum a_{2 n+1}^{(k)}, \sum b_{2 n+1}^{(k)}$ are convergent, and that their sums are $>0$ and $<0$, respectively. That is, there are finite numbers $h^{(k)}>0$, $g^{(k+1)}<0$ such that

$$
\lim _{n} h_{n}^{(k)}=h^{(k)}, \lim _{n} g_{n}^{(k+1)}=g^{(k+1)}, \quad(k=0,1,2, \cdots) .
$$

Turning now to the polynomials $G_{k}(z), H_{k}(z)$ we find by (30) and (31) that for small values of $k$ they converge, for $n=\infty$, to limiting forms which are polynomials of degree depending upon $k$, with positive coefficients if $k>0$, and with negative coefficients if $k<-1$. This same conclusion can be reached for all values of $k$ by virtue of the recursion formulas (12), (13), and (22), in view of the relations (29). We shall express this result as follows:

$$
\begin{array}{ll}
\lim _{n} G_{2 p}=-\beta_{2 p}, & \lim _{n} H_{2 p}=-\alpha_{2 p}, \\
\lim _{n} G_{2 p+1}=\alpha_{2 p+1}, & \lim _{n} H_{2 p+1}=\beta_{2 p+1}, \\
& (p=0, \pm 1, \pm 2, \pm 3, \cdots),
\end{array}
$$

where the $\alpha_{k}(z), \beta_{k}(z)$ are polynomials in $z$. Furthermore by (32)

$$
\begin{aligned}
\beta_{2 p}(0) & =-1, & \alpha_{2 p}(0) & =0 ; \\
\beta_{-2 p-2}(0) & =-g^{\prime}, & \alpha_{-2 p-2}(0) & =1 ; \\
\beta_{2 p+1}(0) & =1, & \alpha_{2 p+1}(0) & =0 ; \\
\alpha_{-2 p-3}(0) & =-1, & \beta_{-2 p-3}(0) & =g^{\prime},
\end{aligned}
$$

Now by (34) and (26) it follows that the expression (28), which is the $n$th approximant in the $k$ th diagonal file of the Padé table for the series (23), converges for $n=\infty$ to a limit $u_{k} / v_{k}$, where

$$
u_{k}=\alpha_{k} p-\beta_{k} p_{1}, v_{k}=\alpha_{k} q-\beta_{k} q_{1} .
$$

These functions satisfy the relation (2) as may be seen with the aid of (27). We state this result in the following theorem. 
THEOREM 1. Let (23) be a series of Stieltjes having a corresponding continued fraction (24) in which $a_{n}>0$. Then if $\sum a_{n}$ converges, the kth diagonal file of the associated Pade table converges to the limit

$$
\frac{\alpha_{k}(z) p(z)-\beta_{k}(z) p_{1}(z)}{\alpha_{k}(z) q(z)-\beta_{k}(z) q_{1}(z)},
$$

where $p(z), p_{1}(z), q(z), q_{1}(z)$ are entire transcendental functions of $z$, and the $\alpha_{k}(z), \beta_{k}(z)$ are polynomials in $z$. If $u_{k}(z), v_{k}(z)$ denote the numerator and denominator, respectively, of the expression (36), then for two indices $k^{\prime}, k^{\prime \prime}$ we have the identity

$$
u_{k^{\prime}} v_{k^{\prime}}-u_{k^{\prime}} v_{k^{\prime}}=\alpha_{k^{\prime}}-\alpha_{k^{\prime}} \beta_{k^{\prime}} .
$$

The writer showed elsewhere that in the case under consideration, $u_{k^{\prime}} / v_{k^{\prime}} \neq u_{k^{\prime \prime}} / v_{k^{\prime}}$ if $k^{\prime} \neq k^{\prime \prime}$ and $k^{\prime}, k^{\prime \prime} \geqq 0$ or $k^{\prime}, k^{\prime \prime} \leqq 0$. We are now able to prove the following supplementary theorem.

TheOREM 2. Under the hypotheses of Theorem 1, if $k^{\prime} \neq k^{\prime \prime}$, then $u_{k^{\prime}} / v_{k^{\prime}} \neq u_{k^{\prime \prime}} / v_{k^{\prime \prime}}$, and therefore the right member of (2) is not identically 0. Furthermore, the functions $u_{k} / v_{k}$ have a pole at $z=0$ if $k \geqq 0$, but are regular at $z=0$ if $k<0$, vanishing there if $k<-1$.

In view of the earlier result mentioned above, it is clearly sufficient to prove here only the final statement in the theorem. But this follows at once from (35) and the equations*

$$
p(0)=-g^{\prime}, \quad p_{1}(0)=1, \quad q(0)=1, \quad q_{1}(0)=0 .
$$

NORTHWESTERN UNIVERSITY

* See formulas for the $P_{n}(z), Q_{n}(z)$ in Stieltjes' memoir, loc. cit. $\S 2$. 